\title{
Virtual Reality Applications in Physical Education Management
}

\author{
Lei Chen \\ Command and Tactical Department of Henan Police College \\ Zhengzhou, Henan 450053 \\ China
}

\begin{abstract}
Virtual Reality as a technology has tremendous potential for application in many fields which help to find, create and innovate. We have identified sports and human health management as one potential area of application. Virtual reality enables the participants in sports to know the techniques in sports and help to update in the domain. This paper presents the current scenario in the application of virtual reality and reviews the developments. Further this work has presented the simulation exercises in the virtual reality.
\end{abstract}

Key words: Virtual Reality, Sports Management, Simulation, VLE

Received: 18 March 2019, Revised 8 June 2019, Accepted 21 June 2019

DOI: $10.6025 / \mathrm{jic} / 2019 / 10 / 3 / 87-90$

(C) 2019 DLINE. All Rights Reserved

\section{Introduction}

With the popularization of "technology Olympics", "Digital Olympics" and "Humanistic Olympics" concept in 2008 and the rapid development of computer technology, Modern science and technology has penetrated into all fields of sports with its powerful affinity, especially plays a tremendous role in sports training. The rapid development of virtual reality technology provides wide space application in competitive sports field. To our country's advantage projects — diving, gymnastics, trampoline, sailing, windsurfing and other projects in the 2008 Beijing Olympic Games, they used virtual reality technology for simulation training in order to realize the sports training methods transformation from traditional human observation to capture and motion analysis of high precision measuring method of human, from approaches based on experience to human motion modeling and Simulation of human motion analysis method. Therefore, this improves the training level and athletic level of our country faster and more effectively in these projects, to ensure that these projects can impact more gold medals in the Beijing Olympic Games, and realize China’s glory plan in 2008 Olympic Games.

\section{Summarize Virtual Reality Technology and Sports Stimulation System}

\subsection{Virtual Reality Technology}

Virtual reality, which also can be abbreviated to VR, stimulates the world of human sense generated by computer. Sometimes are 
called the virtual environment. VR technology embodies the characteristics of multi-subjects, and is a multi-disciplinary complex art accompanying fast development of computer technology, computer graphics, computer network technique, picture processing, pattern recognition, intelligent interface technology, physiology, multi sensor technology, speech processing and audio technology and so on. The operator can produce be personally on the scene through the visual, auditory, olfactory, tactile, and interaction, which can provide new media for human-computer interaction. Nowadays, VR technology has been widely used in military simulation, fire simulation, entertainment, games, medicine, remote control robot, virtual design, virtual manufacturing and other fields.

\subsection{Sports Stimulation System}

Sports stimulation system is interdisciplinary including comprehensive sociology, management, sports training, computer science, graphics, prediction, psychology, math and so on. It is a integrated experimental science with the method of system simulation research on sports issues. It analyzes the problems in sports field through the method of system analysis, and uses related knowledge about math, graph theory, gray theory, operational research, control theory, information theory and so on to establish simulation pattern, then conducts real time, super real-time, under real-time simulation demonstration by using computer technology and connecting graphics, film and video BA and psychology. Finally, it is a typical multi- subjects, multi-factor analysis and comprehensive integration of the disciplines comprehensive disciplines and evaluated, planned, decision-maked by experts. It reproduces the training experience and intention of the coach, organization schemes of the managers and the training process of athletes by computer simulation technology to explain, analyze, predict, organization and evaluate sports education system. In that way, it is a strategic technology to promote sports progress. Athletes need many feel in sports training (visual, auditory, tactile and olfactory etc), and the simulation of virtual reality technology emphasizes a variety of perceptions, interaction and immersion. Therefore, with the further development of virtual reality technology, the simulation training with virtual reality technology will be widely used in the field of sports.

\section{Sports Systematic Simulation Based on VR}

\subsection{Sports Simulation System Based on VR}

Sports simulation system based on VR uses VR technology to do simulative training of competitive sports. It provides effective training tools for new coach and athletes, not only to rapidly improve the effect of exercise training and technology content, but also expend the scope of application of the system simulation, and promote the rapid development of VR technology. Therefore, VR has promising prospect in sports simulative training, and that depend on traits of VR. VR system has variety output form (picture, sound, character and so on). The ability of dealing with a variety of input devices can perform collision detection, realtime interaction, viewpoint control and complex behavioral modeling. In sports training, the use of VR technology can use many kinds of perception to present coach's training intention or athlete's training process, and the coaches and athletes can naturally interact with this simulation system. Application of this kind of physical simulation system based on VR will further improve the scientific training level of sports.

\subsection{Development Status of Competitive Sports System Simulation Based on VR}

- Development status of competitive sports system simulation based on VR, VR has been widely used in the military simulation, fire simulation, medical and many other fields, its purpose is in certain circumstances (hostage negotiation, parachute, firefighting etc. ) and simulative training on students. This training allows the wrong, but not brings any risk and harm to the practitioners. Compared with other methods of training, sports training simulation based on VR have a variety of perception, therefore it can enhance the ability of athletes and sports simulation system for interactive, improve the effect of exercise training. At present, the virtual environment has less application study in competitive sports training, this is mainly because the requirements of competitive sports training to the performance of the VR system are higher than the virtual game, such as the requirements for user friendly interactive, real-time, high precision and strong sense of immersion, in addition to the virtual reality software, hardware technology. Conditions (such as virtual reality special interaction is more expensive equipment, the existing interaction is not convenient and flexible system), real time and accuracy are to be improved; application and popularization of these constraints limit the VR technology in the field of competitive sports training simulation. U.Y. Yang et al. realized a immersive training system based on virtual reality technology, whose core idea is a metaphor for "intuitionist ghost based on interactive method", being called "Just Follow Me". In the training process, trainer action is real-time visualization, and the visualization image training is equivalent to the ghost out of trainee' body through intuitionist ghost metaphor. To observe the trainee's movement from different point of view, the trainees (or as quickly as possible) follow the ghost master (trainers'). SeongmhiBaek et al. give reference movement for any trainee adjustment, and provide reference motion effect analysis for 
trainee and take swordsman training as an example to verify the arithmetic. Nowadays, Also it has made a number of research results in simulation and analysis of 3D human motion in China, has successfully developed a VHTrampdineJ71 software system of computer aided trampoline sports scientific training to prepare for the Beijing Olympic Games. The software is different from the color image analysis based on physical training software, which is based on the digital 3-D human motion simulation technology, human biomechanics data and real human motion data, the 3D simulation, design, analysis and action, therefore, practice has stronger directive significance to sports training.

- Based on the development status of VR in competitive sports simulation, VR technology has the specific scope of application in competitive sports simulation are as follows: the scientific monitoring sports training; sports training information collection, analysis system; advanced training technology application demonstration; training and equipment, equipment; a variety of gymnastics editing. These research results will be applied widely in trampoline, diving, gymnastics and other competitive sports training. So competitive sports simulation based on VR has a broad development prospect.

\subsection{Function of Competitive Sports System Stimulation Based on VR}

- Structure training scene and equipment. According to specific requirements of the specific sports on training scenario building virtual training scenarios, such as, sailing and windsurfing training time is subject to certain conditions (for instance, the appropriate wind speed, wave height and weather), this system constructs realistic training environment field data the system through collecting a variety of sensors and mathematical models of real driving 3D visual, and meets the non training condition in this environment (such as no waves without wind conditions) to complete the training mission. It also can make the virtual training and simulation human, such as, gymnastics athletes can be invited to connect virtual gymnasium in virtual instruments and see how to change the shape of the gymnastics equipment. This technique not only can be used in daily training, but also in the adjustment period before the game to make the athletes play out of their own level in the race.

- Capture exercise date. Through the sensor tracking equipment, it is directly recorded motion data and used to generate computational motorized painting. The biggest advantage of this method is able to capture the athletes (including training apparatus) real exercise data. Due to the generation of movement is basically movement "copy" of a player (instruments), the effect is very generally real. According to the captured motion data, it can edit, modify, design new action, let the coaches to design their own mind "ideal" action, in order to ensure the scientific training. This function can be used wildly in weightlifting, gymnastics, trampoline, narrow water project.

- Collecting physiology and biochemistry and mental date. Physiological, biochemical and physical index is an important indicator of the State Sports. According to different athletes, the biochemical, physiological and psychological data of athletes can be collected through a variety of sensors and intelligent instrument. The collection of physiology includes mainly obtaining pulse, blood pressure and so on which is human metabolism index, all sorts of organ and system function index. According to the collection index, coach can rearrange and redesign action to meet function and characteristics of this project. And coach can pick out action which need arrange with the intention, therefore, coach choose the best arrangement to make sure scientific and reasonable training methods.

- Repeat the Performance and Show the Movement. Movement reappearing is the key function of sports simulation system. Traditional camera shooting can't realize it in some condition. When study on the innovation of new movements in gymnastics project, athletes can realistically and accurately reproduce the gymnastics athletes with a variety of action sports simulation system with its 3D virtual gymnastics to help assistant trainers and athletes innovate action, improve the technical level.

\subsection{System Composition}

The sports simulation system has three parts: input system, output system, virtual environment generator. The input system includes date glove, location orientation tracker, transverter, and glove input transverter etc. Virtual environment generator has custom system, simulation supervisor, three-dimension processor, high performance computer, graphic card, three-dimension model date, touch and force sense feedback device and HD ports etc. The output system contains signal converter and effect converter, so that the user can feel more real and natural of the stereo vision, stereo sound, and completely immersed in the virtual environment, like be personally on the scene.

\section{Conclusion}

With the 2008 Olympic Games held in Beijing, it will promote China's in-depth study of competitive sports simulation technology

Journal of Intelligent Computing Volume 10 Number 3 September 2019 


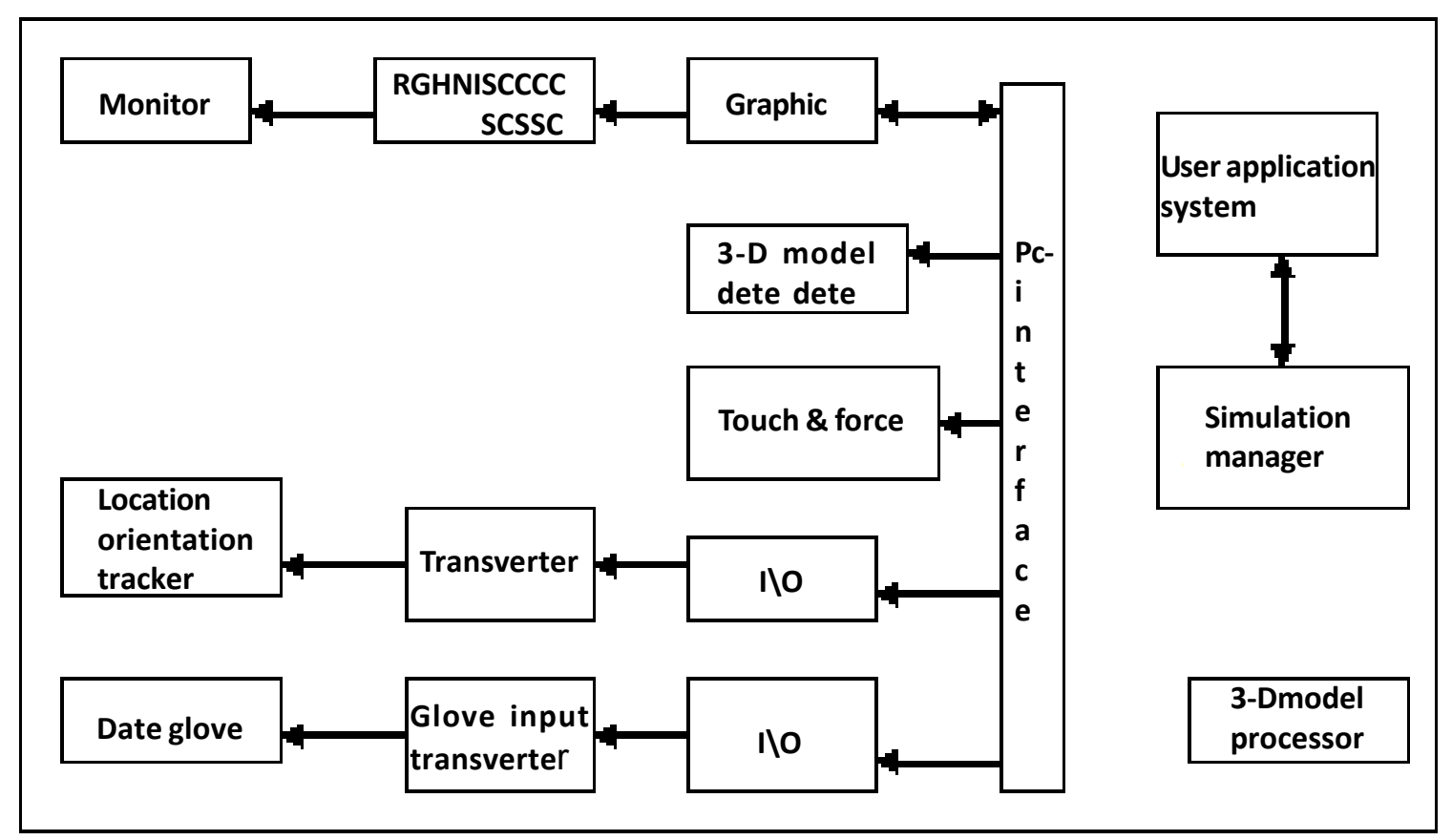

based on VR. Along the development of virtual reality technology in competitive sports in the simulation of widely used will further enhance the technology content of the sports athletics level and the training process. Through the analysis, simulation technology will play an increasingly important role based on VR in sports training, competition, recovery, material process and competition environment. Therefore, the development of dynamic and trace of VR technology, is of great practical significance to study the development of VR technology in competitive sports.

\section{References}

[1] Progress in computer simulation of 3-D digital human motion, CAS Bulletin 2004.

[2] Wang, Chengwei., Gao, Wen., Wang, Xingren. (1996). Theory, Pratice and application of VR technology, Tsinghua University Press.

[3] Li, Xiangchen., Sun, Jinhai. (2001). Sports Simulation System. Beijing: Peoples Sports Publishing House.

[4] Yang, U. Y., Kim, G. J. (2002). Implmentation and Evaluation of “Just Follow me. Aiiinunersive* vr — based, motion training systey. Presence: Tdeoperators and Virtual Environments, 11 (3) 304 - 323, (June).

[5] Seongmin, Baek., Seungyoug, Lee., Kim Gerard Joughyun. (2001). Motion Evaluation for VR — based Motion Training, EUROGRAPHICS, 20 (3) 2001.1_10.

[6] Yan, Min., Zhou, Chuanshan., Wang, Pandeng. (1999). Science and Sports. Beijing: Popular Science Press.

[7] Andy, Sykes. (2003). News Virtual gym fixes it for young athletes, http://www. shu. ac. uk. //schools/slm/news/ sportl, html.

[8] Meng, Guangyun., Li, Xiaoping., Li, Pengfei. (1995). Sports Science and New Technology in the Future, Chinese Sports Science and Technology Journal.

[9] Ji, Qingge. (2003). Mass Virtual Choreography and Prototype System Research. Zhejiang: Zhejiang University Postdoctoral Research Report 2003-9-11.

[10] Pan, Zhigeng., Jiang, Xiaohong. Summarize Distributed Virtual Environment, Journal of Software.

\section{Author Biography}

About the author: Lei Chen (1974-) is associate professor from Xuchang city of Henan, Han nationality. He is a doctor whose research direction police tactics. His address is Railway Police College, No 31, Nongye Road, Zhengzhou, Henan (postcode: 450053). 